\title{
Displacement and force control of a quarter car using a mixed mode MR mount
}

\author{
Shuo Wang ${ }^{\mathrm{a}, *}$, Mohammad Elahinia $^{\mathrm{a}}$ and The Nguyen ${ }^{\mathrm{b}}$ \\ ${ }^{a}$ Dynamic and Smart Systems Laboratory, The University of Toledo, Toledo, OH, USA \\ ${ }^{\mathrm{b}}$ MIE Department, University of Minnesota Duluth, Duluth, MN, USA
}

Received 21 December 2011

Revised 24 March 2012

\begin{abstract}
In hydraulic hybrid vehicles (HHV), vibration in dual-mode pump/motor units should be isolated from the chassis. A mixed mode magnetorheological (MR) fluid mount was adopted to isolate this vibration and was evaluated in a quarter car model. The MR fluid mount was designed to be able to operate in flow mode and squeeze mode independently and simultaneously. For HHVs, it is desirable to control force and displacement transmissibility. These simulation results presented a basis for designing an effective algorithm to control both the displacement transmissibility and force transmissibility. Moreover, a hierarchical controller for minimizing the two requirements for transmissibility was also constructed. At last, a fuzzy logic controller was devised to closely reproduce the effect of the hierarchical controller. The experiments were set up to facilitate the hardware-in-the-loop evaluation of the mount. Results from the experiments showed that the mixed mode MR fluid mount was able to achieve desired dynamic stiffness profile to minimize the dual-transmissibility criterion.
\end{abstract}

Keywords: MR mount, HHV, hardware in the loop test, displacement control, force control, fuzzy logic control, hierarchical control

\section{Nomenclature}
$I_{i}: \quad$ fluid inertia
$k_{r}$ : $\quad$ stiffness of the upper rubber part
$\dot{x}_{i}$ : the fluid velocity through the flow path
$b_{r}$ : damping of the upper rubber part
$A_{i}$ : $\quad$ cross-sectional area of the flow path
$R: \quad$ radius of the squeeze plate
$R_{i}$ : the fluid resistance at zero magnetic field
$F_{\text {exc }}: \quad$ excitation force
$C_{1}$ : compliance of the top chamber
$k_{u}$ : $\quad$ unsprung stiffness
$C_{2}$ : compliance of the bottom chamber
$b_{u}$ : $\quad$ unsprung damping
$L: \quad$ length of the flow path
$M_{c}$ : mass of chassis
$h$ : distance between the magnetic poles
$H_{-} F$ : field for flow mode

\footnotetext{
${ }^{*}$ Corresponding author: Shuo Wang, Dynamic and Smart Systems Laboratory, The University of Toledo, Toledo, OH, USA. E-mail: petershuowang@gmail.com.
} 


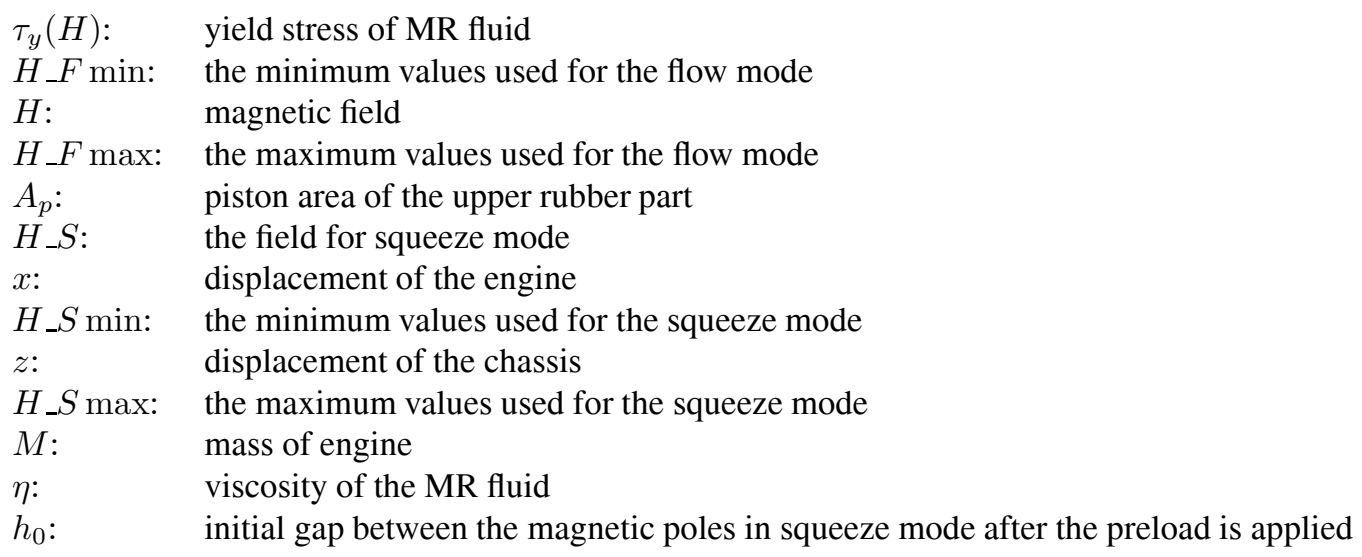

\section{Introduction}

Solutions to the noise, vibration and harshness (NVH) issues have always been the interest in many fields especially the automotive industry for improving the ride and handling characteristics. Vehicle frame is constantly subject to both external excitation sources such as road condition and internal ones, i.e. engine or other power sources, when a vehicle is moving on the road. Suspension system is designed to eliminate the vibration transmitted from the road to the chassis. Meanwhile, engine mounts are required for dealing with vibration transmitted from the engine or the pump/motor of hydraulic hybrid vehicles (HHV). Many devices have been developed to prevent vibration transmission which could be passive, active or semi-active. Passive mounts are not able to adjust their damping and stiffness to meet the vibration isolation requirements. Active mounts can achieve good vibration isolation, but they require substantial external energy to operate the actuators and supply the control forces. Semi-active control systems can adapt the damping and the stiffness without inputting much energy into the system. MR fluid-based devices belong to semi-active category. The MR fluid dampers are designed to reduce the vibration transmitted from the road to the chassis, while the MR fluid mounts are invented for mitigating the vibration from the engine or pump/motor to the chassis.

Design and modeling the MR fluid mount has drawn great attention to provide desired dynamic stiffness over a range of working frequency. Ahn et al. designed one of the earliest magnetorheological mount. The mount uses inertia track in a conventional hydraulic mount as the valve that opens or closes the MR fluid flow from upper chamber to the lower chamber and it works as the flow mode [1]. Ha et al. presents a squeeze mode type MR mount. The MR fluid filled the gap between the two cores and was enclosed by a strip of natural rubber. When the upper electromagnetic core and lower electromagnetic core are close enough, squeeze reaction force is generated by the fluid [2]. Researchers are attracted to incorporate multiple working modes since each single mode has its own limitations. Choi et al. proposed a shear and flow mixed mode MR mount [3]. Brigley et al. presented an MR vibration isolator which is claimed to work in all three modes [4]. However, questions could be raised to these attempts. By the definition of the three working modes, it is challenging to explain the simultaneity of flow mode and shear mode. Also these designs were not able to identify the contribution of each mode. Nguyen et al. by Dynamic and Smart System Laboratory proposed a novel MR mount incorporating the flow working mode and squeeze working mode induced by separate electromagnetic coils [5]. The mount has been modeled, fabricated and experimentally verified. The control methodology in this paper is designed to be applied to this mixed mode MR fluid mount. Compared to previous designs in other papers, the effects of each working mode and combined working mode for the MR fluid mount can be clearly identified and investigated.

Various control algorithms for MR fluid-based devices have been researched and proposed so that these semi-active devices can achieve satisfactory performance for vibration isolation. Unsal et al. developed the skyhook control for MR dampers with the advantages of selective energy dissipation for a model of 6 DOF (degrees of freedom) parallel platform [6]. The control algorithm reduces the peak at the first resonant frequency and decreases the transmissibility at higher frequency at high frequencies. Koo et al. proposed on-off groundhook (on-off DBG) control to effectively 
reduce the vibrations of the primary structure using an MR tuned vibration absorber [7]. The study showed that the controlled TVA outperformed a passive system, the resonant peaks reduced and good isolation around the structure's natural frequency. Ahmadian et al. developed a control method combining fuzzy logic and skyhook/groundhook for the semi-active MR system to improve the overall performance for the vehicle heave and roll motion during vehicle maneuvers [8]. The results show that this fuzzy logic control for the semi-active suspensions could greatly reduce the vehicle heave and roll displacements while it increases the body acceleration peak value for road inputs at the tire which cause a less comfortable ride. Wang et al. presented the modeling and control of MR fluid dampers using neural networks to deal with the inherent nonlinear behavior of MR fluid [9]. The simulation results are satisfactory which provide a new method for the damper controller of the MR fluid damper. However, it requires training. The training data needs to be able to cover most situations of practical applications and also needs to be simple in order to speed up the training process. Furthermore, the neural networks works like a "black box" as the process of generating the outcome is not explicitly stated. An RD-1005-3 MR damper by Lord Corporation is used by Ali et al. for their study of a base isolation system for a three-storey building. The Bouc-Wen model is used and dynamic inversion based control and integrator backstepping based control are stated and compared with clipped optimal control and optimal fuzzy logic control [10]. From the results, the dynamic inversion based control and integrator backstepping based control are better than clipped optimal control and genetic algorithm based fuzzy logic control because the latter two controllers decreased the isolator displacement at the cost of an increase in superstructure acceleration while the former two controllers provide a trade-off between isolator displacement and superstructure acceleration. However, there are few papers focusing on the control of MR fluid mounts. A linear quadratic Gaussian (LQG) controller was designed by Hong et al. for a MR fluid mount to isolate the vibration of a structural system [11]. The acceleration levels of structural system are attenuated effectively by controlling the damping of MR mount. The force transmission through the two rubber mounts is also suppressed by activating the MR mount. However, the results show that acceleration and force transmission with the LQG control are higher than the one without control at higher frequencies. Differences exist between the MR fluid dampers and MR fluid mounts in their modeling and design, therefore the control of MR fluid mounts requires better attention.

Transmissibility, dynamic stiffness, damping value, Phase, and coherence are important in characterizing the performance of engine and transmission mounts. Among these indicators, displacement transmissibility [6,7,12] and force transmissibility [13] are the most popular index to display the performance of the vibration isolation. Control algorithms are designed to target realizing the lowest values. Most papers focus on the analysis of one type transmissibility: either displacement transmissibility or force transmissibility. Research on both transmissibilities is seldom seen and the how to minimize both requires more attention. This paper studied the behavior of a mixed mode MR fluid mount used in a two DOF model based on quarter car concept. Both displacement transmissibility and force transmissibility are examined for the effect of flow mode and squeeze mode separately and simultaneously and skyhook control on flow mode and squeeze mode separately and simultaneously. The simulation results show that the combined skyhook control for both modes can achieve the better vibration isolation than control of one mode. However, the lowest possible displacement transmissibility and force transmissibility by skyhook control do not happen under common conditions. Addressing this issue, a hierarchical controller was constructed for realizing the tradeoff between these two types of transmissibility. Furthermore, a fuzzy logic controller is devised to closely reproduce the effect of the hierarchical controller. The experiments are set up to realize the hardware-in-the-loop tests. Results from the experiments show that the mixed mode MR fluid mount is able to achieve desired dynamic stiffness which is directly related to vibration transmissibility.

\section{Modeling}

A two degree of freedom (2DOF) model based on quarter car concept is built to investigate the effect of the MR fluid mount for preventing the vibration transmitted from engine to the chassis. Because the study focuses on the vibration isolation ability of the MR fluid mount, the suspension and tire (unsprung components) are simplified as a damper and a spring in parallel as shown in Fig. 1. The excitation is assumed to be a force profile acting on the engine. 


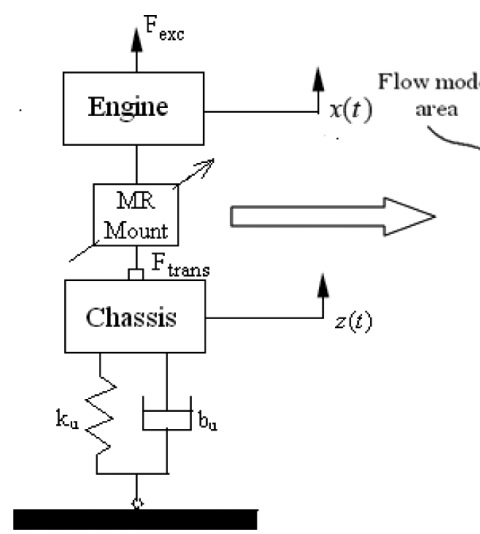

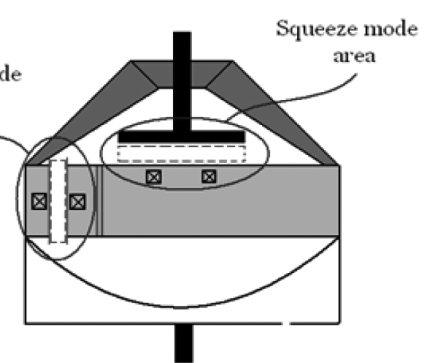

(b)

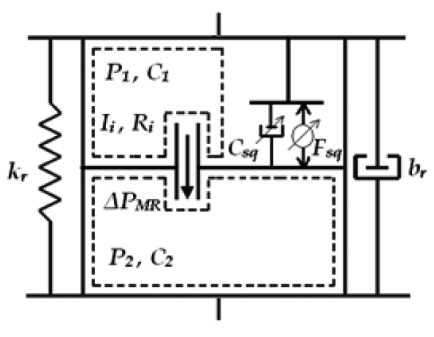

(c)

(a)

Fig. 1. (a) Configuration of two DOF based on quarter car concept using MR fluid mount (b) schematic, (c) physical model.

The mixed mode MR fluid mount model also shown in Fig. 1 has two working modes: flow mode and squeeze mode [5]. They are induced by separate electromagnetic coils so that the effects of each mode and the combined mode can be investigated. When the MR fluid travel from one chamber to the other through the flow path, the outer coil is powered to produce the magnetic field; the flow mode is on. The activation of the inner coil can hinder the motion of the rod which makes the squeeze mode active. The stiffness and damping are therefore adjusted by the activation of these two working modes. Beside the hydraulic structure in the design, the MR fluid elements are filled in which constitutes an MR fluid mount.

The governing equations characterizing the system were derived as:

$$
\begin{aligned}
& I_{i} A_{i} \ddot{x}_{i}+R_{i} A_{i} \dot{x}_{i}+A_{i}\left(\frac{1}{C_{1}}+\frac{1}{C_{2}}\right) x_{i}+2 \frac{L}{h} \tau_{y}(H) \operatorname{sign}\left(\dot{x}_{i}\right)=\frac{A_{P}}{C_{1}}(x-z) \\
& M \ddot{x}+\left(b_{r}+C_{s q}\right)(\dot{x}-\dot{z})+\left(k_{r}+\frac{A_{p}^{2}}{C_{1}}\right)(x-z)+F_{s q}-\frac{A_{i} A_{p}}{C_{1}} x_{i}=F_{e x c} \\
& \left(b_{r}+C_{s q}\right)(\dot{x}-\dot{z})+\left(k_{r}+\frac{A_{p}^{2}}{C_{1}}\right)(x-z)+F_{s q}-\frac{A_{i} A_{p}}{C_{1}} x_{i}-k_{u} z-b_{u} \dot{z}=M_{c} \ddot{z}
\end{aligned}
$$

Where $C_{s q}=3 \eta \pi R^{4} / 2\left(h_{0}+(x-z)\right)^{3}$ and $F_{s q}=4 \pi R^{3} \tau_{y}(H) \operatorname{sign}(\dot{x}) / 3\left(h_{0}+(x-z)\right)$ are the effects of the squeeze mode according to Hong et al. [14].

The meaning of these parameters appeared in the equations of motion are shown in nomenclature.

The transmitted force can be represented by:

$$
F_{\text {trans }}=\left(b_{r}+C_{s q}\right)(\dot{x}-\dot{z})+\left(k_{r}+\frac{A_{p}^{2}}{C_{1}}\right)(x-z)+F_{s q}-\frac{A_{i} A_{p}}{C_{1}} x_{i}
$$

Both the force transmissibility and displacement transmissibility can be investigated since the excitation force, transmitted force, and engine displacement, chassis displacement are either known or calculable. The force transmissibility is defined as the ratio of the transmitted force divided by the excitation force as Eq. (5). The displacement transmissibility is defined as the ratio of the displacement of the chassis divided by the displacement of the engine as Eq. (6).

$$
\begin{aligned}
& T R_{-} f=F_{\text {trans }} / F_{\text {exc }} \\
& T R_{-} d=z / x
\end{aligned}
$$

The system of Eqs (1) (2) and (3) are constructed in MATLAB/Simulink ${ }^{\circledR}$ to obtain the response of MR fluid mount. Furthermore, the skyhook controller is designed on the basis of the modeling of the MR fluid mount. 


\subsection{Skyhook control}

Skyhook control is one of the most popular control methods for MR fluid devices. The governing equations can be represented as:

$$
\begin{cases}\dot{x}(\dot{x}-\dot{z})>0 & \text { Field On } \\ \dot{x}(\dot{x}-\dot{z})<0 & \text { Field Off }\end{cases}
$$

Where

$\dot{x}=$ absolute velocity of the engine

$\dot{x}-\dot{z}=$ relative velocity between the engine and the chassis

When the absolute velocity of the engine and the relative velocity between the engine and the chassis have the same direction, the magnetic field is turned on. When they have the opposite direction, the magnetic field is turned off.

This paper investigates the effect of skyhook control of flow mode and squeeze mode separately and simultaneously. For the skyhook control on flow mode only, the control law is

$$
\begin{cases}\dot{x}(\dot{x}-\dot{z})>0 & H_{-} F=H_{-} F \max \\ \dot{x}(\dot{x}-\dot{z})<0 & H_{-} F=H_{-} F \min \end{cases}
$$

For the skyhook control on squeeze mode only, the control law is

$$
\begin{cases}\dot{x}(\dot{x}-\dot{z})>0 & H_{-} S=H_{-} S \max \\ \dot{x}(\dot{x}-\dot{z})<0 & H_{-} S=H_{-} S \min \end{cases}
$$

For the skyhook control on simultaneous flow and squeeze mode, the control law is

$$
\left\{\begin{array}{cc}
\dot{x}(\dot{x}-\dot{z})>0 & H_{-} F=H_{-} F \max , H \_S=H \_S \max \\
\dot{x}(\dot{x}-\dot{z})<0 & H \_F=H_{-} F \min , H \_S=H \_S \min
\end{array}\right.
$$

\section{Simulation results}

Simulations have been executed in the MATLAB/Simulink ${ }^{\circledR}$ to investigate the effect of the flow mode and the squeeze mode separately and simultaneously for both displacement transmissibility and force transmissibility. Based on these results, skyhook control for the flow mode and squeeze mode as well as the combined mode were also performed to observe the effectiveness of the control law. The excitation level for the system is $100 \mathrm{~N}$ from the engine as it shows as $F_{\text {exc }}$ in Fig. 1(a). The MR fluid mount has its working frequency range from $0 \mathrm{~Hz}$ to $100 \mathrm{~Hz}$. The noisy data observed in some simulation results are the result of numerical solver limitation.

Figure 2 represents the displacement transmissibility and force transmissibility without activating either the flow mode or the squeeze mode. The MR fluid mount works as a hydraulic mount based on its modeling [5]. The significant changes in transmissibility take place in $0-20 \mathrm{~Hz}$ for both the displacement transmissibility and the force transmissibility. The peak and notch of these two kinds of transmissibility happened at different frequencies. The force transmissibility shows two peaks due to its involving with the movement of both engine and chassis. The displacement transmissibility shows one peak because the ratio decouples the movement of the engine and chassis.

The effect of MR fluid mount and skyhook control law are investigated for the displacement transmissibility and force transmissibility respectively.

\subsection{Force transmissibility}

Figures 3-7 demonstrate the simulation results for the force transmissibility. Figure 3 shows the effect of flow working mode. The activation of flow mode does not decrease the first peak significantly but evidently contribute to the decrease of the second peak. The extra damping is supplied when the magnetic field for the flow mode is turned on which drops the peak value. The stiffness is also increased due to the field which results in a higher resonance frequency. Increasing the field continuously lead to the solidification of the MR fluid inside the flow path and the 


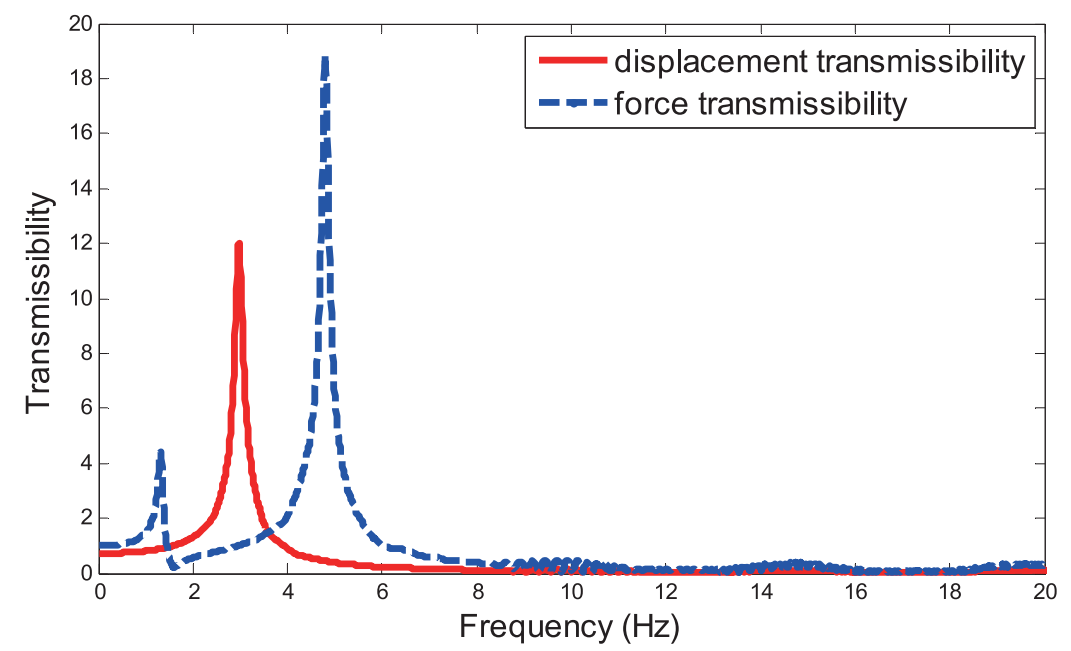

Fig. 2. Displacement transmissibility and force transmissibility without fields.

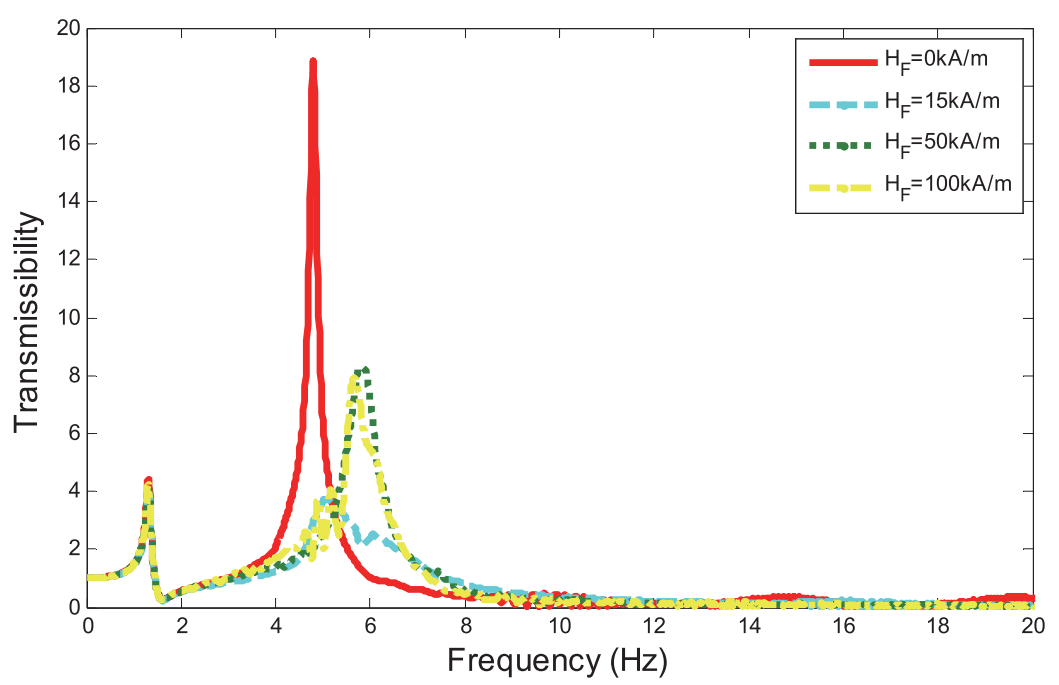

Fig. 3. Effect of flow mode on force transmissibility.

damping reduces which results in the increase of the peak again. Figure 4 shows the effect of the squeeze mode. The activation of the squeeze mode reduces both peaks when the magnetic field for the squeeze mode is set to a small value. The effect of squeeze mode is more obvious than that of the flow mode to some extent. However, the further increase of the field for squeeze mode causes a uniform increase of the transmissibility within the entire working frequency range. This may be caused by the solidification of the MR fluid around the squeeze plate.

Figures 5 and 6 show the transmissibility plot when skyhook control law is applied to the flow mode and squeeze mode individually. Based on the simulation results of effect of each mode, the values of the applied fields for the skyhook control are chosen. The field value is chosen which can produce the lowest transmissibility between the transmissibility without field and the one with field. For example, the transmissibility follows the one with the field of $50 \mathrm{kA} / \mathrm{m}$ before the crossover point and the one without field after the crossover point in Fig. 5. Both figures demonstrate that the skyhook control can track the lowest transmissibility for the whole working frequency range. The skyhook method on flow mode decreases the second peak and shifts the resonant frequency to a higher value. The skyhook on squeeze mode decreases the first peak and makes the second peak disappear. From the observation of these two figures, skyhook control on squeeze mode provides better vibration isolation compared to the skyhook control on flow mode at lowering the peak transmissibility value. 


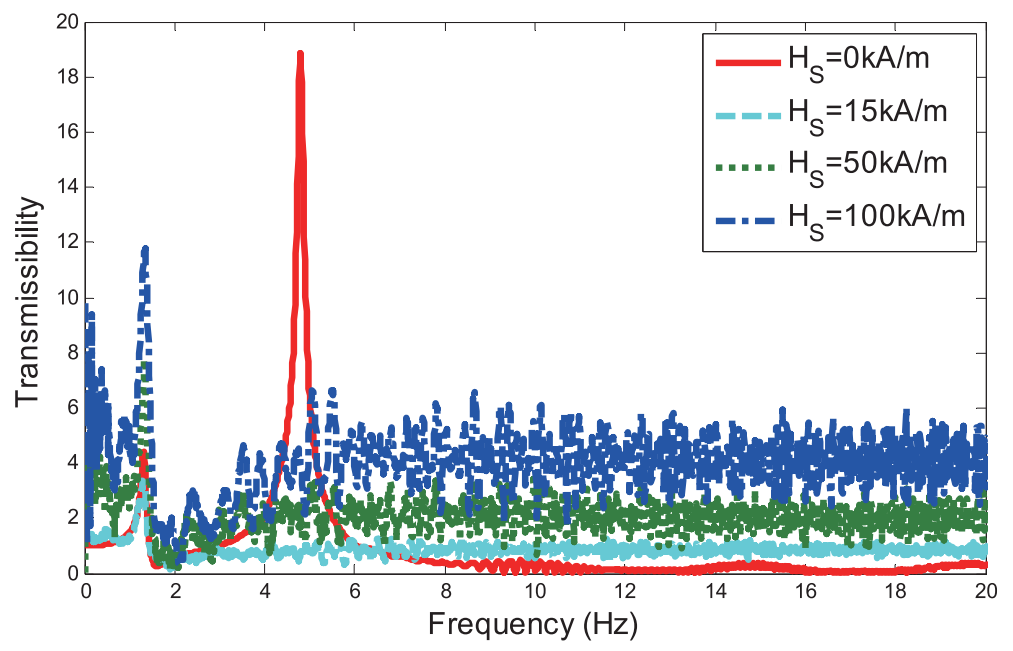

Fig. 4. Effect of squeeze mode on force transmissibility.

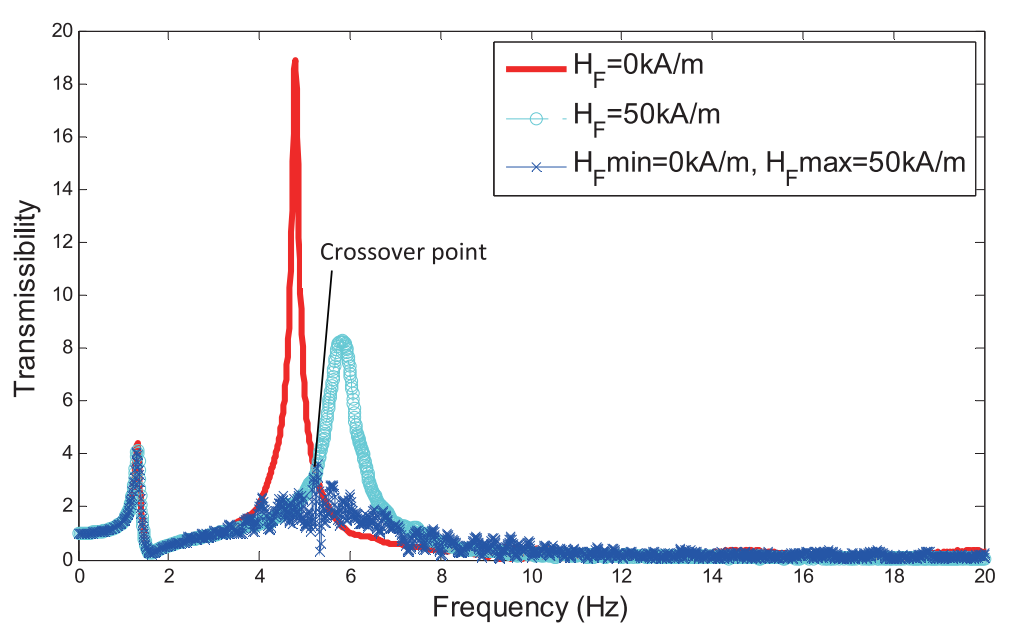

Fig. 5. Skyhook control on flow mode for force transmissibility.

Figure 7 shows the combined skyhook control on both flow mode and squeeze mode. The skyhook control on either mode can certainly reduce the transmissibility. However, the combined skyhook control can provide slightly better isolation though the difference between squeeze mode and combined mode is minor. It is also observed that when both the flow mode and squeeze mode are activated and controlled simultaneously, the squeeze mode exhibits a more influential effect in contributing to the decrease of transmissibility. It can be concluded that to achieve the lowest force transmissibility by skyhook control, the magnetic field for flow mode and squeeze mode should be set to $50 \mathrm{kA} / \mathrm{m}$ and $15 \mathrm{kA} / \mathrm{m}$.

\subsection{Displacement transmissibility}

Figures 8-12 demonstrate the simulation results for the displacement transmissibility. Similar to force transmissibility, the investigations for the effect of the flow mode and squeeze mode without any control, skyhook control on flow mode and squeeze mode individually as well as on the combined mode have been conducted. These simulation results show the similar pattern compared to those of the force transmissibility. For Fig. 11, the transmissibility is viewed in the logarithmic scale which makes it clear that the skyhook control for on squeeze mode can obtain lower values at higher frequencies than the one without control. It can be concluded that to achieve the lowest displacement 


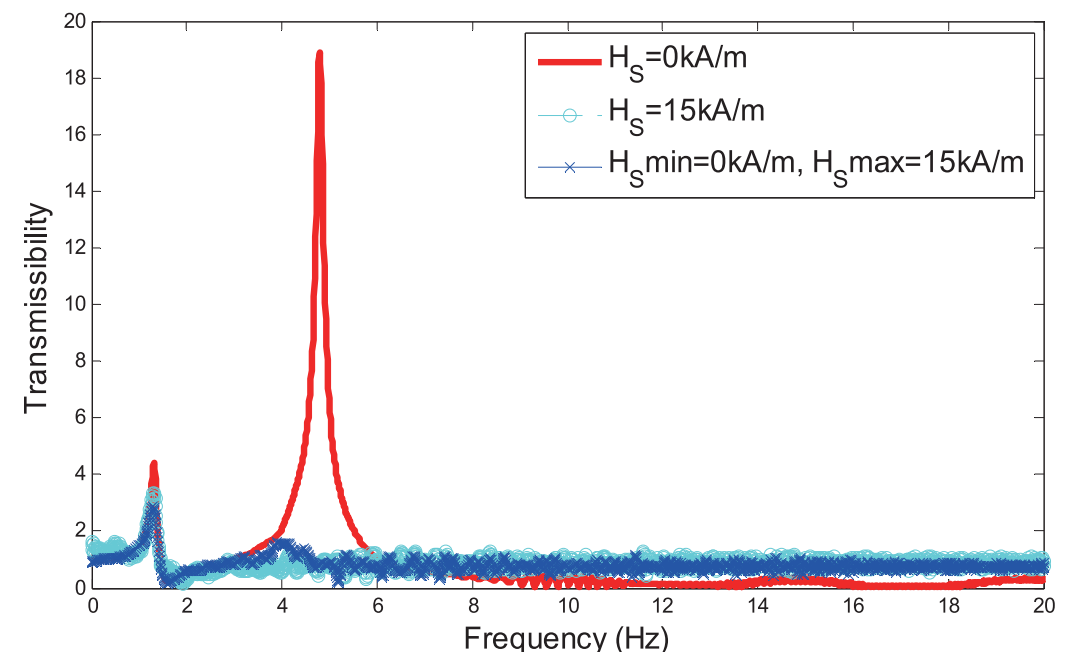

Fig. 6. Skyhook control on squeeze mode for force transmissibility.

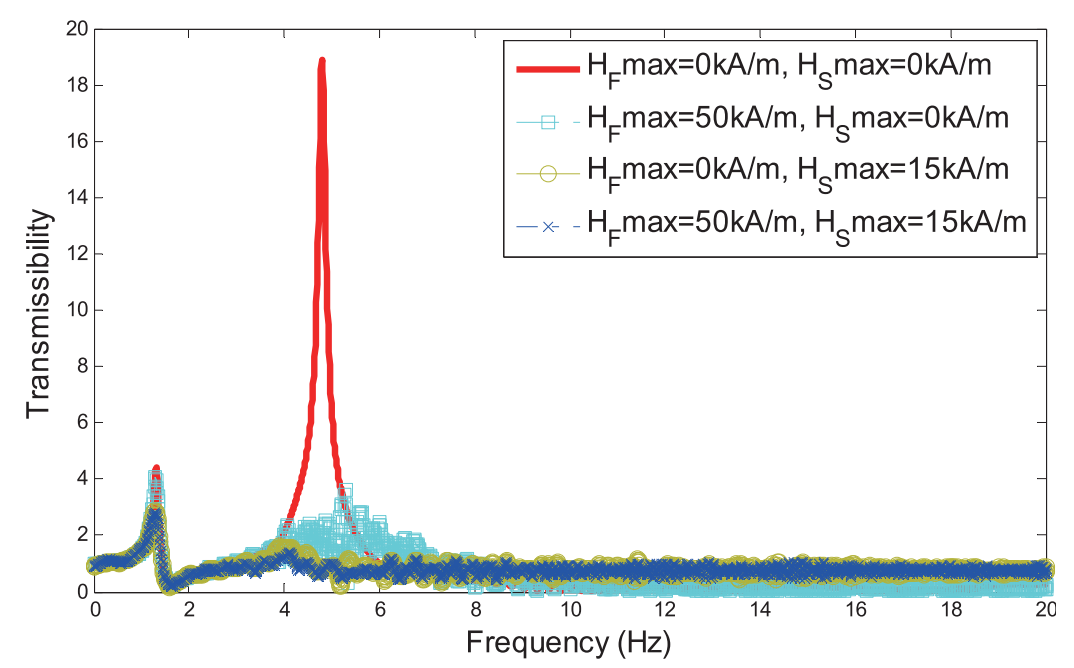

Fig. 7. Skyhook control on both modes for force transmissibility.

transmissibility by skyhook control, the magnetic field for flow mode and squeeze mode should be set to $30 \mathrm{kA} / \mathrm{m}$ and $5 \mathrm{kA} / \mathrm{m}$.

From the simulation results of Figs 7 and 12, it is not difficult to discover that the conditions to achieve the lowest displacement transmissibility and force transmissibility by skyhook control are not consistent with each other (flow mode $\max 50 \mathrm{kA} / \mathrm{m} \&$ squeeze mode $\max 15 \mathrm{kA} / \mathrm{m}$ vs. flow mode max $30 \mathrm{kA} / \mathrm{m} \&$ squeeze mode max $5 \mathrm{kA} / \mathrm{m}$ ). So these two types of transmissibility cannot obtain the lowest values at the same time. There exists a trade-off between these two types of transmissibility if the skyhook controller is insisted. The further research therefore involves finding a controller than can minimize both transmissibilities in simulation followed by experimental evaluation.

\section{Hierarchical controller design in simulation}

The hierarchical control designed to realize the tradeoff between the lowest displacement transmissibility and force transmissibility is realized by switching to the proper values. The principle is to use the magnetic field values to maintain the lowest displacement transmissibility. Whenever the acceleration is over certain value, the other set 


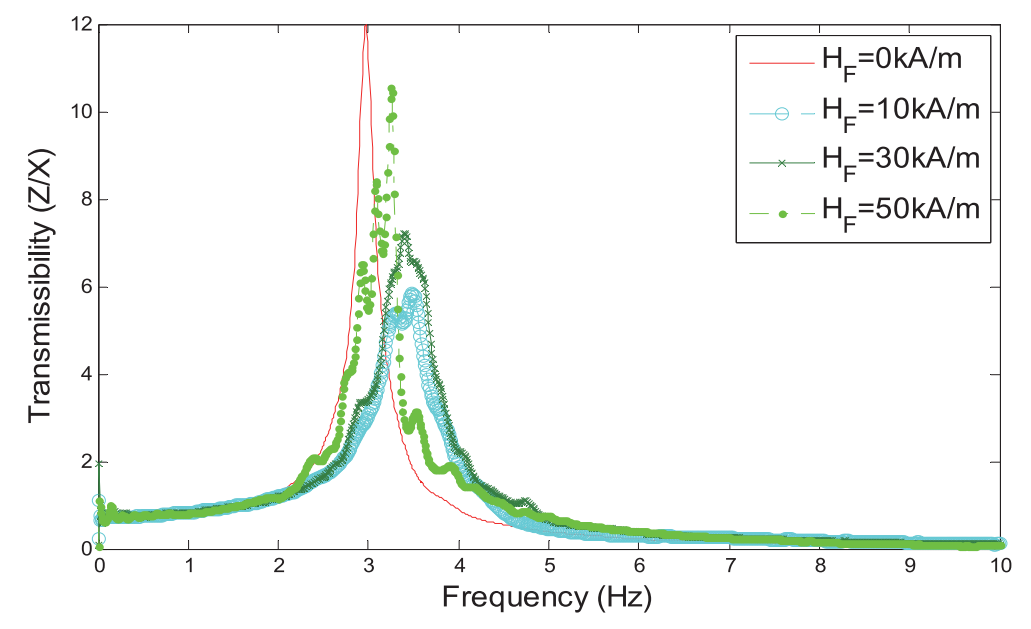

Fig. 8. Effect of flow mode for displacement transmissibility.

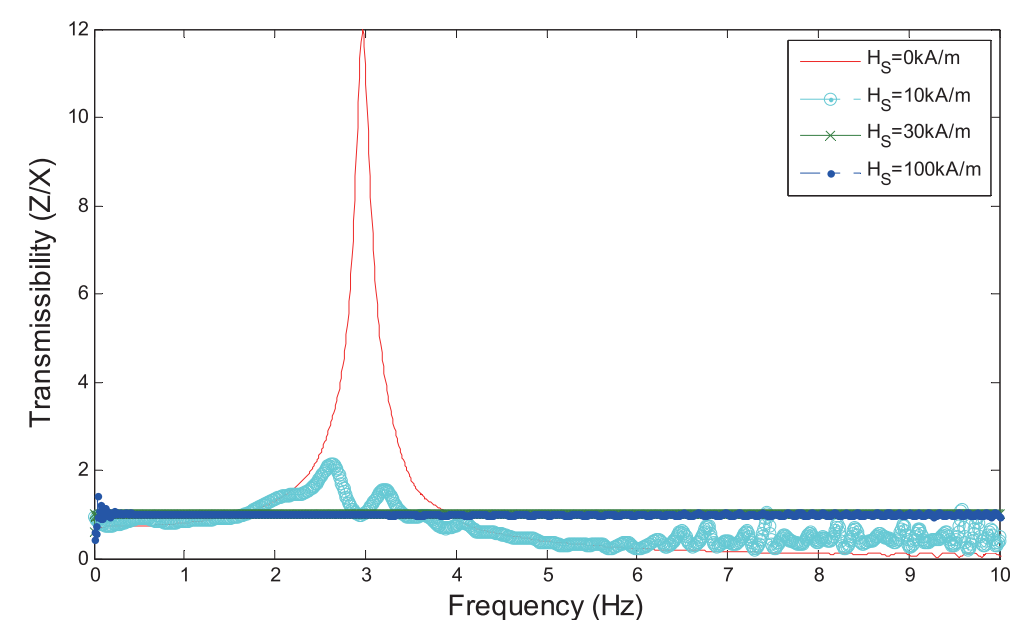

Fig. 9. Effect of squeeze mode for displacement transmissibility.

of field values are employed to maintain the lowest force transmissibility. If the acceleration decreases to be smaller than that certain value, the set of field values to achieve lowest displacement transmissibility will be reinstated.

The Simulink ${ }^{\circledR}$ model of hierarchical control of the MR mount in two degree of freedom structure has been built as shown in Fig. 13. There are four main components in the model: excitation, suspension and chassis, MR mount and engine, and controller.

Inside the Excitation block, there are two kinds of excitation: the displacement excitation from the road and the force excitation from the engine (or pump/motor). It depends on the simulation need to choose which excitation will be used or if both excitations are required. In this paper, only the force excitation form the engine is used. In the Suspension and Chassis block, the suspension is simplified as a damper and a spring. The chassis can be influenced by displacement excitation and the reaction force created by MR fluid mount. The chassis displacement, velocity and acceleration are the outputs of this block. In the MR mount and Engine block the MR mount model is the same as the one in previous sections. The inputs of this block are the magnetic field for flow mode and squeeze mode, and the force excitation, while the outputs are the transmitted force, the relative velocity between the chassis and the engine, and the velocity of the engine. The Controller block takes chassis acceleration, relative velocity between chassis and engine and engine velocity as inputs and produces the appropriate magnetic field for flow mode and squeeze mode. The controller is a hierarchical control with two layers: the basic layer control is to switch between 


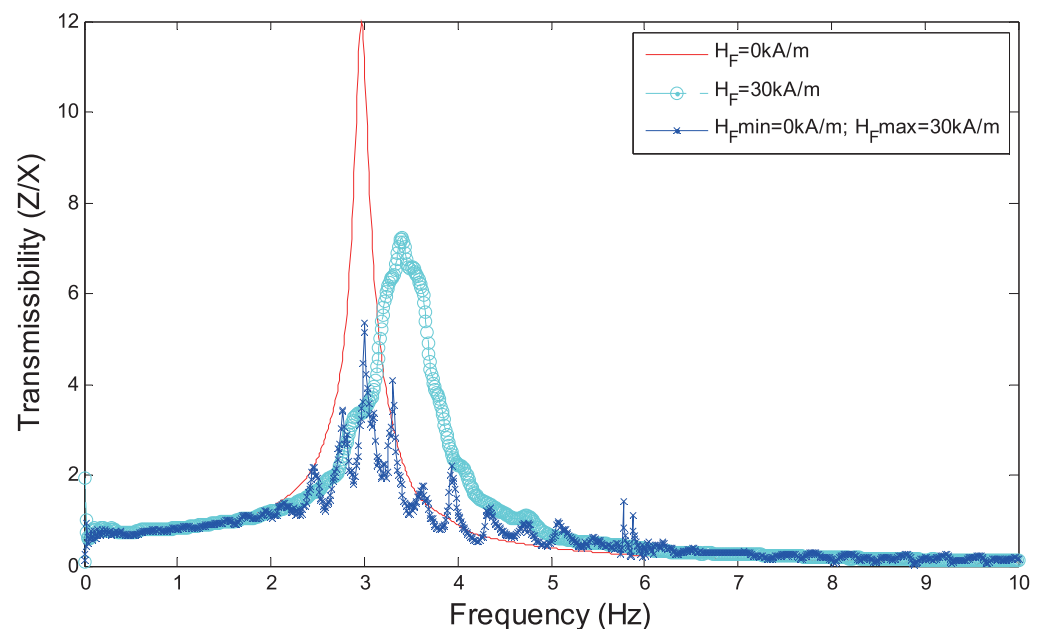

Fig. 10. Skyhook control on flow mode for displacement transmissibility.

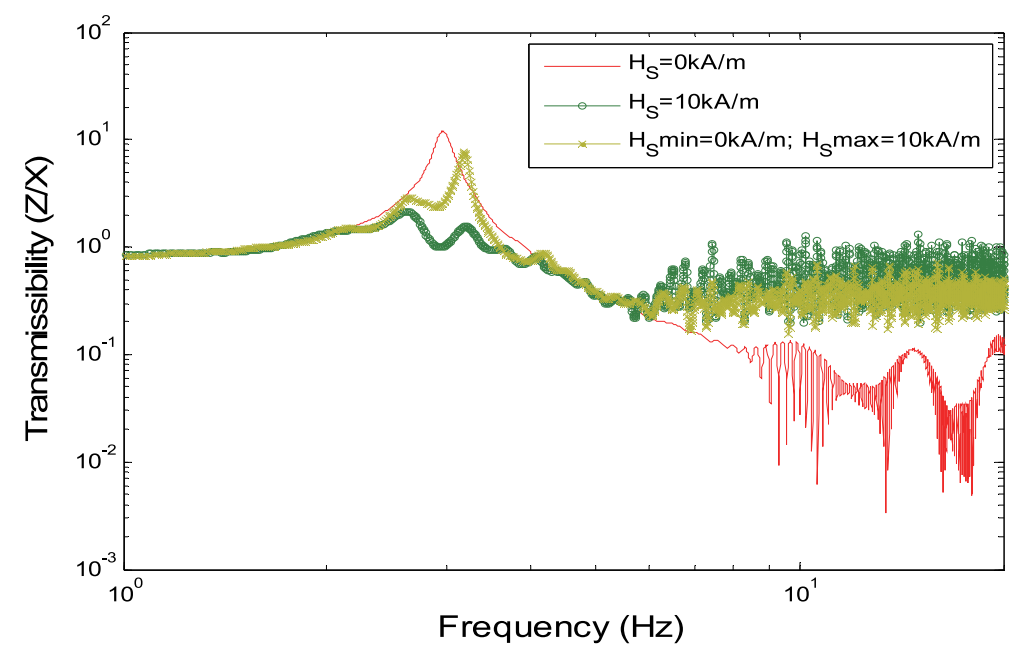

Fig. 11. Skyhook control on squeeze mode for displacement transmissibility.

the maximum magnetic field value and the minimum magnetic field value (decided by the skyhook algorithm); the second layer control is switch between the lowest force transmissibility and lowest displacement transmissibility.

With the hierarchical control design, the simulation result can be observed in Fig. 14. The lowest displacement transmissibility is pursued as the primary law. Whenever the acceleration of the chassis is over $0.2 \mathrm{~m} / \mathrm{s}^{2}$, the values to achieve the lowest force transmissibility are implemented. If the acceleration value falls under $0.2 \mathrm{~m} / \mathrm{s}^{2}$, the values to achieve the lowest displacement transmissibility are chosen to be used again. The switch acceleration value is chosen based on observed simulation result. The acceleration values are under $0.3 \mathrm{~m} / \mathrm{s}^{2}$, the $0.2 \mathrm{~m} / \mathrm{s}^{2}$ is chosen to protect the chassis from vibrating acceleration higher than that. The significant decrease in both displacement transmissibility and force transmissibility can be observed and at the same time the trade-off has been realized.

For the two layers of hierarchical control, the skyhook control in first layer switch values of between maximum and minimum to achieve the lowest transmissibility (either displacement or force); the control in the second layer switch the sets of values between the lowest displacement transmissibility and lowest force transmissibility. Every switch in the system makes the running of the system unsmooth because this logic causes oscillation between two values. From the control perspective, it requires time to reach certain values and also may cause "overshoot". This is unpleasant feature of switch logic. Fuzzy logic control, on the other hand, is known for provision of smooth control surface. Therefore, a fuzzy controller has also been designed to imitate the effect of the hierarchical controller. 


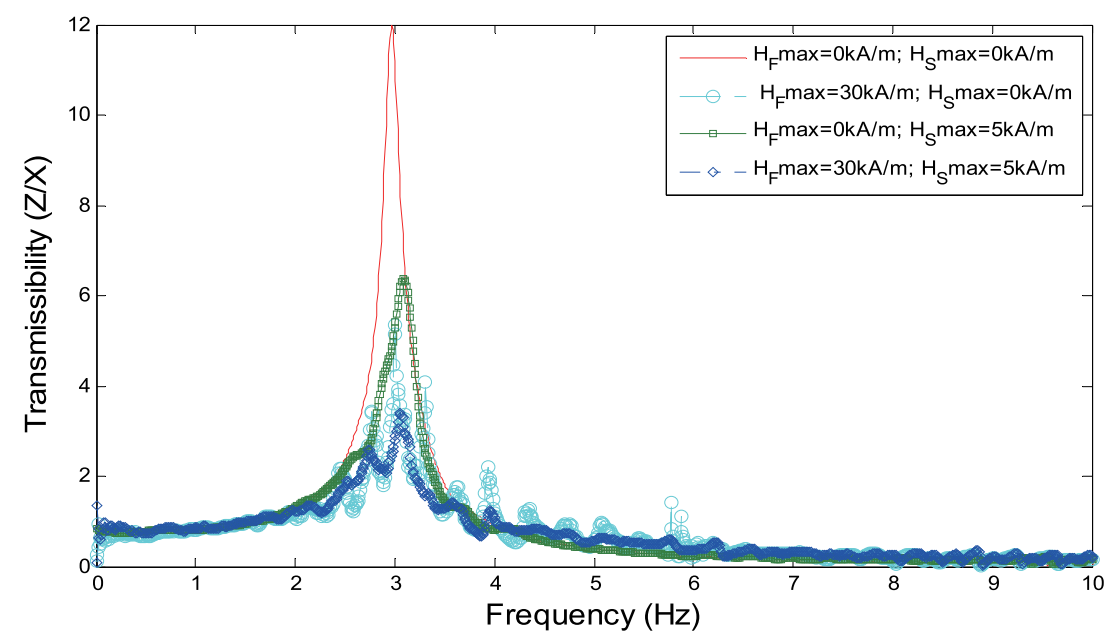

Fig. 12. Skyhook control on both modes for displacement transmissibility.

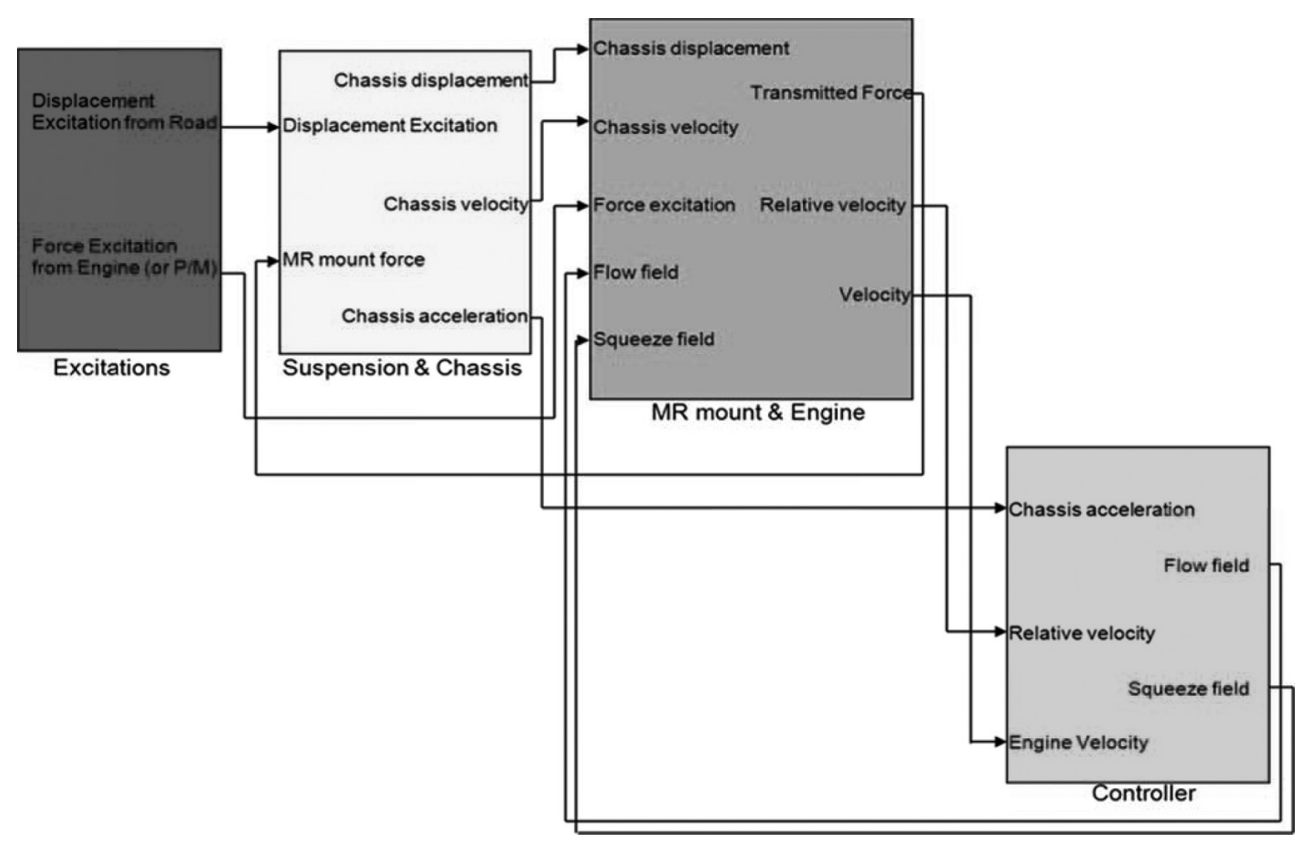

Fig. 13. Model of hierarchical control of the MR mount in 2DOF.

The Fuzzy Logic Toolbox in Simulink is used to intelligently choose the value for magnetic field for flow mode and squeeze mode. The control algorithm is programmed in the Fuzzy Inference System (FIS) Editor. Membership Function Editor inside the FIS Editor is where the input and output membership functions are defined. The Rule Editor inside the FIS Editor is where the rules are added. The model adopted in the controller is Mamdani type. Three inputs for the FIS are relative displacement, relative velocity between the base and suspended mass, as well as acceleration. The output for FIS is magnetic field. The gauss membership functions are used for both inputs and outputs.

The simulation results between these two controllers are shown in Fig. 15. Three sets of transmissibility are shown: the force transmissibility and displacement transmissibility without any field (the last two on the legend), the ones with hierarchical control (the first two on the legend) and the ones with fuzzy logic control (the middle two on the legend). As it appears, the fuzzy logic controller can also achieve similar results for both displacement and 


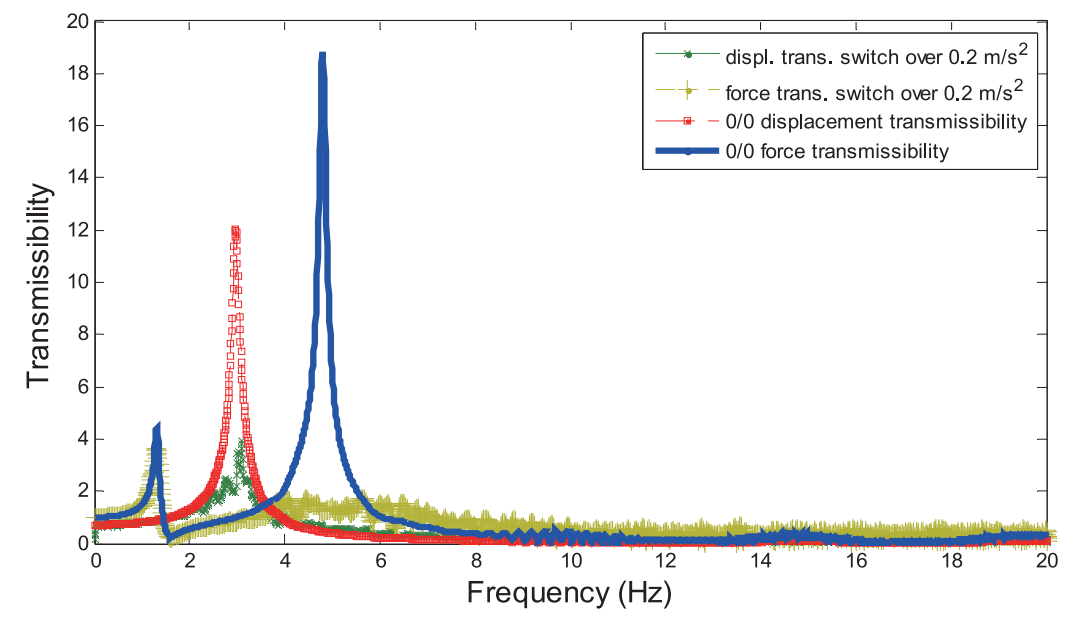

Fig. 14. Tradeoff between two types of transmissibility.

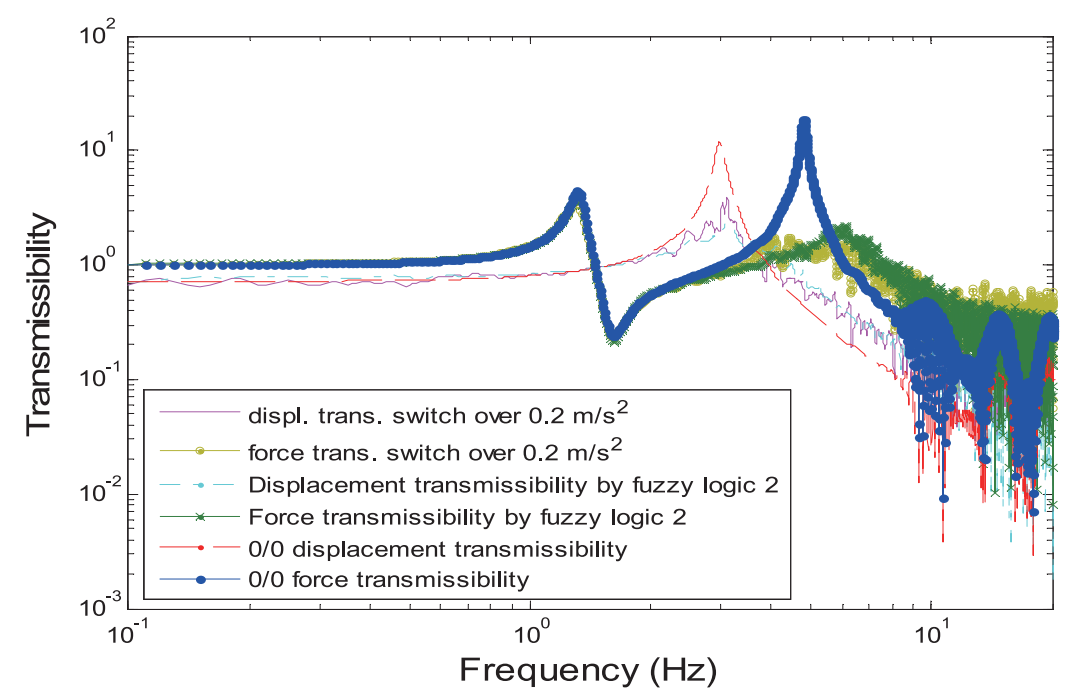

Fig. 15. Comparison between two controllers: fuzzy logic and hierarchical.

force transmissibility. Compared to the hierarchical controller, fuzzy logic controller shows better performance at higher frequencies. However, at lower frequencies, the hierarchical controller can provide slightly better vibration isolation. During the design of the fuzzy logic controller, neither the concept of displacement transmissibility nor force transmissibility is considered, but they can achieve similar results of the hierarchical control. Also, the control surfaces are smooth and continuous. However, the rule development requires professional knowledge of certain area, as in this paper it needs some foreknowledge of how much field value should be used in the rule design. This foreknowledge can be obtained by observing the simulation results of hierarchical control.

\section{Hardware in the loop tests}

By the definition of dynamic stiffness and transmissibility [5]:

The calculation of dynamic stiffness: 


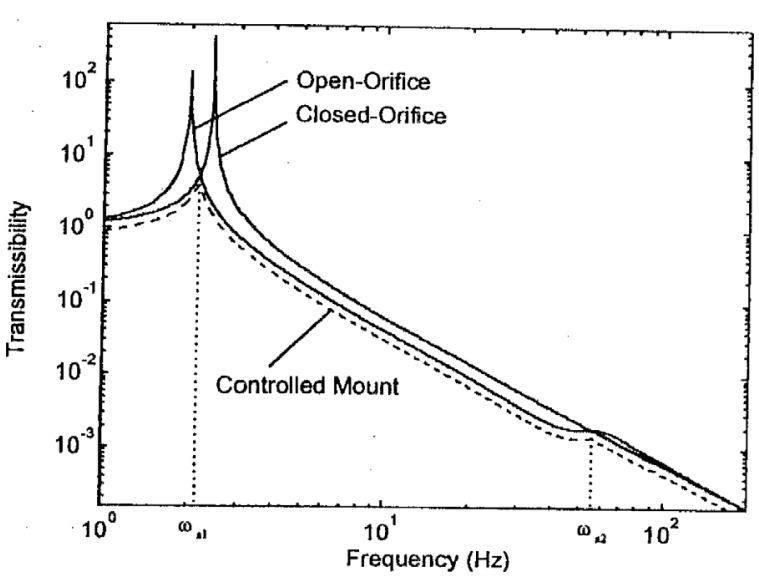

Fig. 16. Transmissibility over frequency [1].

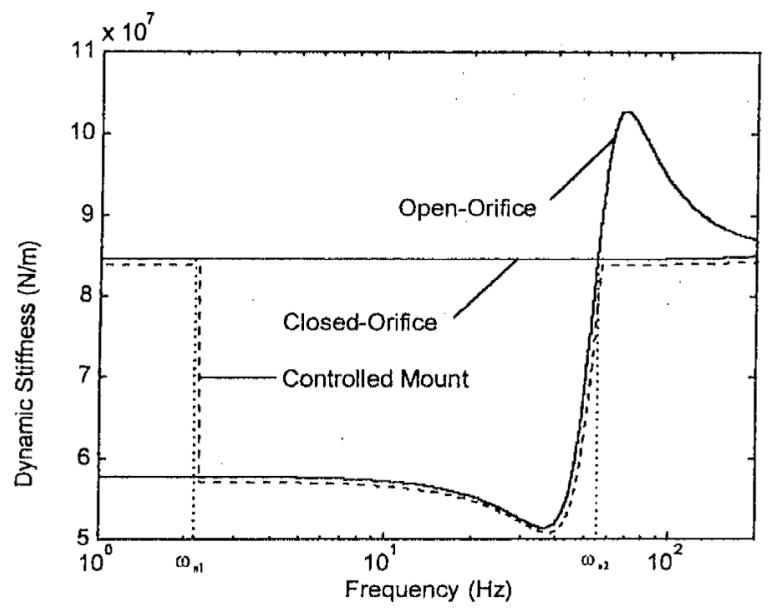

Fig. 17. Dynamic stiffness over frequency [1].

Displacement Excitation $x(t) \rightarrow X(\omega)=F F T(x)$

Transmitted Force $\quad f(t) \rightarrow F(\omega)=F F T(f)$

Dynamic Stiffness $\quad K_{d y n}(\omega)=\frac{F(\omega)}{X(\omega)}=A+i B$

Amplitude $=\left|K_{\text {dyn }}\right|=\sqrt{A^{2}+B^{2}} ;$ Phase $=\tan ^{-1}\left(\frac{B}{A}\right)$

The calculation of transmissibility:

$\begin{array}{ll}\text { Force Excitation } & f_{\text {in }}(t) \rightarrow F_{\text {in }}(\omega)=F F T\left(f_{\text {in }}\right) \\ \text { Transmitted Force } & f_{\text {out }}(t) \rightarrow F_{\text {out }}(\omega)=F F T\left(f_{\text {out }}\right) \\ \text { Transmissibility } & T R(\omega)=\frac{F_{\text {out }}(\omega)}{F_{\text {in }}(\omega)}=C+i D \\ \text { Amplitude }=|T R|=\sqrt{C^{2}+D^{2}} ; \text { Phase }=\tan ^{-1}\left(\frac{D}{C}\right)\end{array}$

As long as a model is built and the excitation is chosen, both the dynamic stiffness and transmissibility can be calculated. Ahn et al. evaluated the performance of a designed magnetorheological mount [1]. The transmissibility of the open and closed valve condition are illustrated in Fig. 16. As it shows, $\omega_{s 1}$ and $\omega_{s 2}$ are the two switching frequencies at which the valve is controlled to be open or closed so that the lowest transmissibility can be obtained. They are also shown in the dynamic stiffness over frequency in Fig. 17. The mount provides a high dynamic stiffness at lower frequencies and a lower dynamic stiffness at a higher frequencies caused by the switching. In other words, a certain dynamic stiffness profile corresponds to a certain transmissibility profile. Therefore, dynamic stiffness is the major indicator of the transmissibility. In the experiments, this figure of merit has been used as a measurement for vibration transmissibility.

As the simulations for the control of MR fluid mount in the single degree of freedom and two degree of freedom have been conducted, one question is left: "Can the desired dynamic stiffness (which corresponds to the lowest transmissibility) be achieved by the prototype mount and testing machine?" If the desired dynamic stiffness at certain frequency can be obtained by the control of the current, it is equivalent to achieve the desired lowest transmissibility at that frequency. This could lead to achieving the desired lowest transmissibility at the entire working frequency range.

The experimental setup is illustrated in Fig. 18:

There are four main parts in this setup. First, ElectroForce ${ }^{\circledR} 3330$ test instrument by BOSE ${ }^{\circledR}$ Corporation is able to generate displacement excitation and measure the transmitted force by a load cell as shown in Fig. 18. By the PCI_82 Control Box, the measurement of the excitation displacement and transmitted force can be extracted and processed.

Second, the displacement and force signals are taken into the dSPACE1104 box, which is an interface between the analog signals and digital signals. There are four columns of the channels, two of them are analog to digital converter channels called ADCH 1-8, and the others are digital to analog converter channels called DACH 1-8. This experiment uses two analog to digital converter channels to input the displacement and force signals (ADCH 5 and 


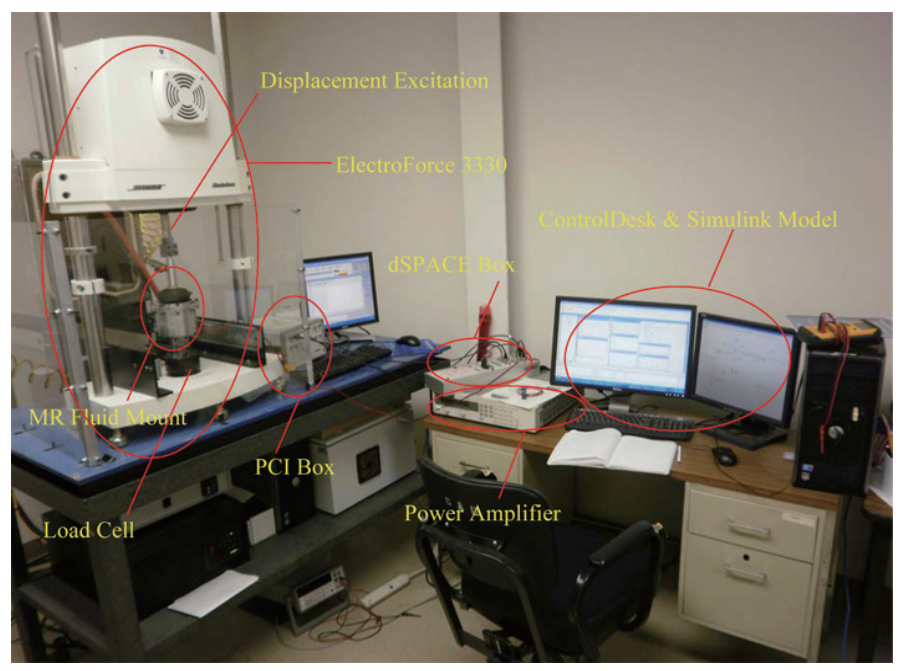

Fig. 18. Experimental setup for the desired dynamic stiffness.

ADCH 6) into MATLAB/Simulink ${ }^{\circledR}$ environment. Also, one digital to analog converter channel (DACH 1) is used to output the desired current the computer calculated to the power amplifier.

Third, the calculated current is not large enough to be supplied by the dSPACE system alone, therefore an Agilent 6543 A DC power supply is also used. The power supply is used in the current control mode such that it amplifies the current values calculated from the computer.

Fourth, the amplified current is output to the appropriate magnetic coil; i.e. either the flow mode coil or the squeeze mode coil. This completes the closed loop control. The ControlDesk by dSPACE and Simulink ${ }^{\circledR}$ by MathWorks ${ }^{\circledR}$ can easily work together by using the dSPACE's Real-Time Interface (RTI). The RTI is the link between the real-time hardware by dSPACE and the Simulink ${ }^{\circledR}$ model from MathWorks ${ }^{\circledR}$. It extends the C code generator Real-Time Workshop ${ }^{\circledR}$ in order that the Simulink ${ }^{\circledR}$ model can be implemented on dSPACE real-time hardware. The ControlDesk test and experiment software can provide virtual instruments, automation and parameter set handling. For a hardware-in-the-loop (HIL) test, dSPACE provides an ideal experiment environment.

The control law adopted here is a proportional control. Based on the envelop values for a certain frequency, a linear increase of the dynamic stiffness is assumed due to the increase of the current. For instance, $20 \mathrm{~Hz}$, the dynamic stiffness without any field is about $255 \mathrm{~N} / \mathrm{mm}$ as a low base line, while the dynamic stiffness with the current 0.4 A for the flow mode is about $380 \mathrm{~N} / \mathrm{mm}$ as a high base line. Therefore the difference of dynamic stiffness $380 \mathrm{~N} / \mathrm{mm}-255 / \mathrm{mm}=125 \mathrm{~N} / \mathrm{mm}$ is caused by $0.4 \mathrm{~A}$. Then a unit increase of dynamic stiffness can be caused by $0.4 / 125 \mathrm{~A}$. For the desired dynamic stiffness, the desired current can be calculated by the operation that the difference between its value and the low base line times the current causing the unit increase of dynamic stiffness. If $350 \mathrm{~N} / \mathrm{m}$ dynamic stiffness is desired to be achieved at $20 \mathrm{~Hz}$, the desired current to realize that will be $(350-255) * 0.4 / 125=$ $0.304 \mathrm{~A}$

\section{Experimental results}

\subsection{Dynamic stiffness - fixed point}

The experiments have been conducted at the frequencies under $30 \mathrm{~Hz}$. Flow mode is chosen to achieve the desired dynamic stiffness (DS). The close match between desired and achieved DS could be observed in Fig. 19.

Compared the experimental dynamic stiffness and desired dynamic stiffness, the error rate is calculated.

$$
\text { Error }=\frac{\mid \text { Desired DS-Experimental DS } \mid}{\text { Desire DS }} \times 100 \%
$$




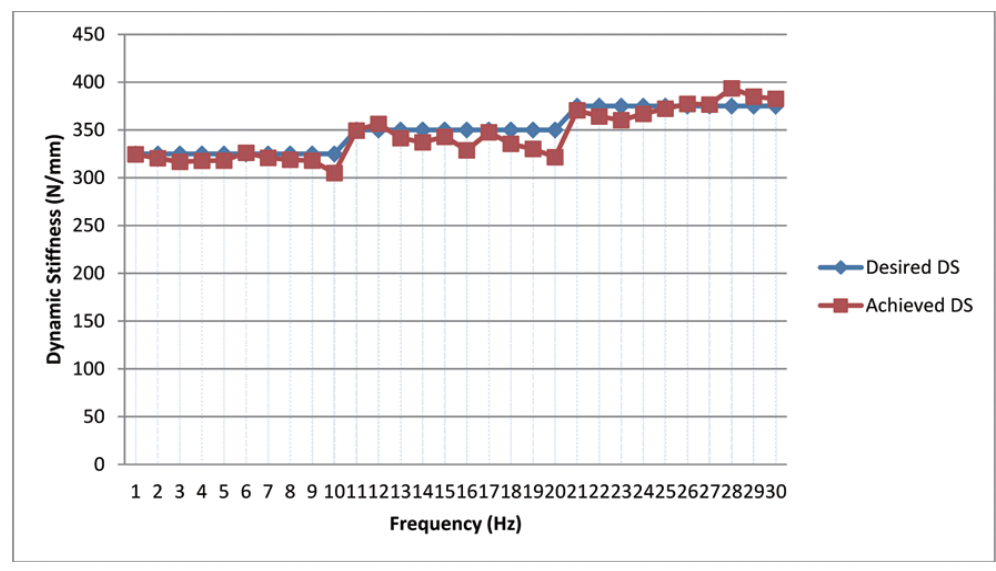

Fig. 19. Comparison between desired DS and achieved DS for fixed points.

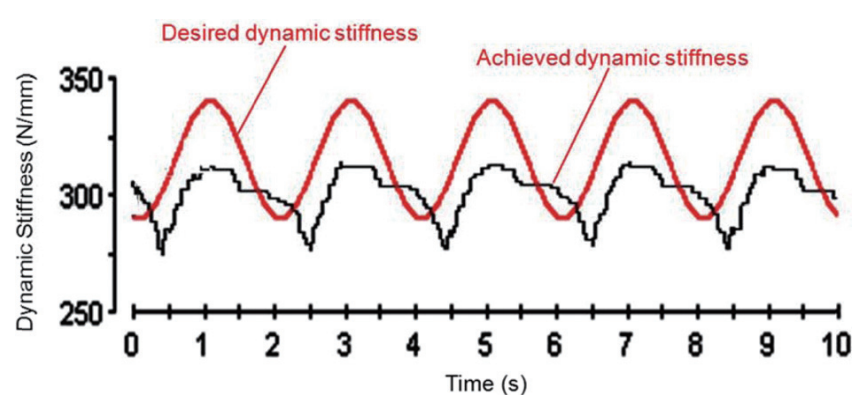

Fig. 20. Desired dynamic stiffness profile.

The largest error that occurred in the tests was $8.12 \%$, and the smallest error was $0.14 \%$. The average error was $2.69 \%$. The error is an indicator of good tracking ability for the desired dynamic stiffness.

As it shows, for each frequency, the desired dynamic stiffness is set; take $24 \mathrm{~Hz}$ for an example, the target is $375 \mathrm{~N} / \mathrm{mm}$, experimental result is about $367 \mathrm{~N} / \mathrm{mm}$. The dynamic stiffness at a certain frequency can be closely achieved. This makes possible the desired dynamic stiffness profile over the whole working frequency. As stated before, the corresponding transmissibility is also achievable which indirectly verified the effectiveness of the controller design in single degree of freedom model and in two degree of freedom model.

\subsection{Dynamic stiffness - variable points}

For sine wave is tested for $10 \mathrm{~Hz}$ to verify that the variable dynamic stiffness can be achieved for certain frequency. The squeeze mode is chosen for this test. The sine wave is

sine wave $=25 * \sin (\pi * t)+315$

The desired dynamic stiffness profile fluctuates around $315 \mathrm{~N} / \mathrm{mm}$ and the fluctuation range is $25 \mathrm{~N} / \mathrm{mm}$ as shown in the layout of ControlDesk software, see the red line in Fig. 20.

The dynamic stiffness achieved is also shown in black. The range of the achieved dynamic stiffness is from about 275 to 315 . The mean value is 295 . The error can be roughly calculated by the following:

$$
\begin{aligned}
& (340-315) / 340 * 100 \%=7.35 \% \\
& (315-295) / 315 * 100 \%=6.35 \% \\
& (290-275) / 290 * 100 \%=5.17 \%
\end{aligned}
$$


The largest error is about $7.35 \%$, the smallest error is about $5.17 \%$, and the average error is about $6.35 \%$. These results are acceptable for the experiment.

This part of experiment proves that the variable dynamic stiffness at a certain frequency can be approximately achieved. It can be inferred that the variable dynamic stiffness can be achieved over a range of frequency. Consider the two degree of freedom model; the lowest displacement transmissibility and lowest force transmissibility correspond to different dynamic stiffness profiles. The experiment show that the switching between two dynamic stiffness profiles can be closed obtained.

In summary, the experiments complete the verification of the model and control of the mixed mode MR fluid mount. It can be concluded by simulations and by experiments that the proposed MR fluid mount is able to provide a certain range of dynamic stiffness over a large range of frequency and also the desired dynamic stiffness could be achieved in reality.

\section{Conclusions}

In this paper, a mixed mode MR fluid mount was introduced. Based on the model, the skyhook control algorithms were designed for flow mode and squeeze mode separately and simultaneously. Both the displacement transmissibility and force transmissibility were investigated. Simulation results indicate that the skyhook control algorithm can obtain the lowest transmissibility for either flow mode or squeeze mode individually. The combined skyhook control for both modes achieved the lowest possible transmissibility over the whole range of the working frequency. However, the displacement transmissibility and force transmissibility reached their minimum by skyhook control under different conditions. The hierarchical control of the MR fluid mount in a two degree of freedom structure was developed to realize the tradeoff between the lowest displacement transmissibility and the lowest force transmissibility. A fuzzy logic controller was also developed to imitate the effect of the hierarchical control system and to provide a continuous control surface at the same time. The modeling of the control is completed in MATLAB/Simulink ${ }^{\circledR}$ and the simulation result shows effectiveness of fuzzy logic control method. Furthermore, the simulation result is compared with the one by the hierarchical controller. Experiments were performed to verify that the desired dynamic stiffness is realizable in order to achieve the optimal transmissibility. Hardware-in-the-loop tests are taken to prove the controllability of the MR fluid mount. Flow mode and squeeze mode have been controlled simultaneously for the whole working range in this paper, the future work can involve control the flow mode and squeeze mode separately for certain working range based on their individual simulation results.

\section{References}

[1] Y.K. Ahn, M. Ahmadian and S. Morishita, On the design and development of a Magnetorheological mount, Vehicle System Dynamics 32 (1999), 199-216.

[2] J.Y. Ha, Y.K. Ahn, B.S. Yang, D.J. Kim and M. Ahmadian, Dynamic properties of squeeze type mount using MR fluid, Proceedings of IMECE 2003, Washington D.C., USA, November 15-21, 2003.

[3] S.B. Choi, S.R. Hong, K.G. Sung and J.W. Sohn, Optimal control of structural vibrations using a mixed-mode magnetorheological fluid mount, International Journal of Mechanical Sciences 50 (2008), 559-568.

[4] M. Brigley, Y.T. Choi and N.M. Wereley, Experimental and theoretical development of multiple fluid mode magnetorheological isolators, Journal of Guidance, Control and Dynamics 31(3) (2008), 449-459.

[5] T.M. Nguyen, A novel semi-active magnetorheological mount for vibration isolation, Dissertation, University of Toledo, 2009.

[6] M. Unsal, C. Niezrecki and C.D. Crane, Six DOF vibration control using magnetorheological technology, SPIE Symposium on Smart Structures and Materials, San Diego, California, USA, February, 2006.

[7] J. Koo, M. Ahmadian and M. Elahinia, Semi-active controller dynamics in a magneto-rheological tuned vibration absorber, Smart Structures and Materials: Damping and Isolation, Proceedings of SPIE, Vol. 5760, 2005.

[8] M. Ahmadian, On the development of fuzzy skyhook control for semiactive magneto rheological systems, Smart Structures and Materials: Damping and Isolation, Proceeding of SPIE Vol. 5760, 2005.

[9] D.H. Wang and W.H. Liao, Modeling and control of magnetorheological fluid dampers using neural networks, Smart Materials and Structures 14 (2005), 111-126.

[10] S.F. Ali and A. Ramaswamy, Hybrid structural control using magnetorheological dampers for base isolated structures, Smart Materials and Structures 18(Issue 5) (2009).

[11] S. Hong and S. Choi, Vibration Control of a Structural System Using Magneto-Rheological Fluid Mount, Journal of Intelligent Material Systems and Structures 16 (2005), 921-936. 
[12] Y.K. Ahn, J.-Y. Ha, Y.-H. Kim, B.-S. Yang, M. Ahmadian, K.K. Ahn and S. Morishita, Dynamic characteristics of squeeze-type mount using magnetorheological fluid, Proc ImechE 219 Part K: J. Multi-body Dynamics, 2005, pp. 27-34.

[13] L.S. Chen, K. Qiu and D.Y. Chen, Research on continuous damping control improving force isolation of a SDOF mounting system, Journal of Intelligent Material Systems and Structures 17 (2006), 347-351.

[14] S.R. Hong, S.B. Choi, W.J. Jung and W.B. Jeong, Vibration Isolation of Structural Systems Using Squeeze Mode ER Mounts, Journal of Intelligent Material Systems and Structures 13 (2002), 421-424. 

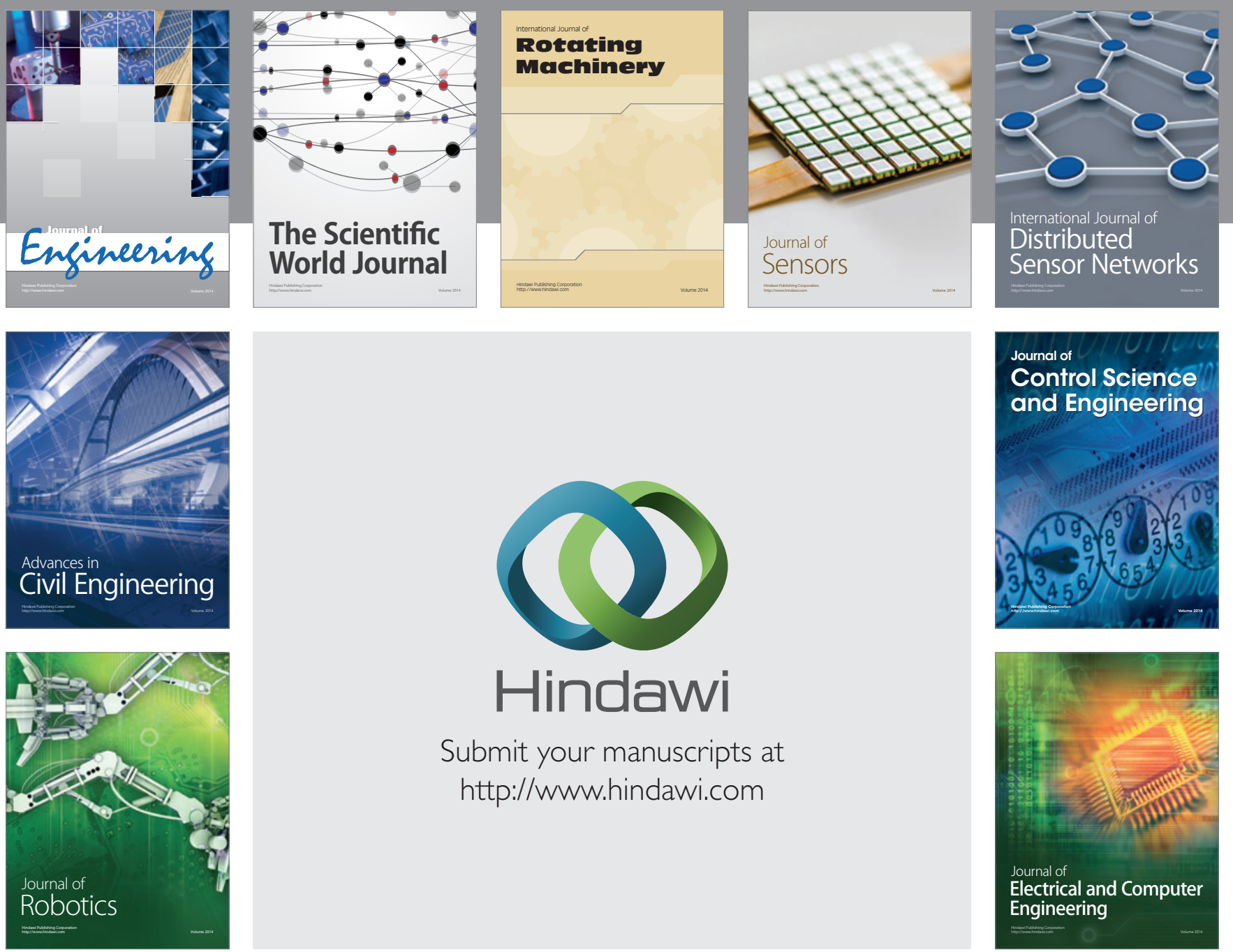

Submit your manuscripts at

http://www.hindawi.com
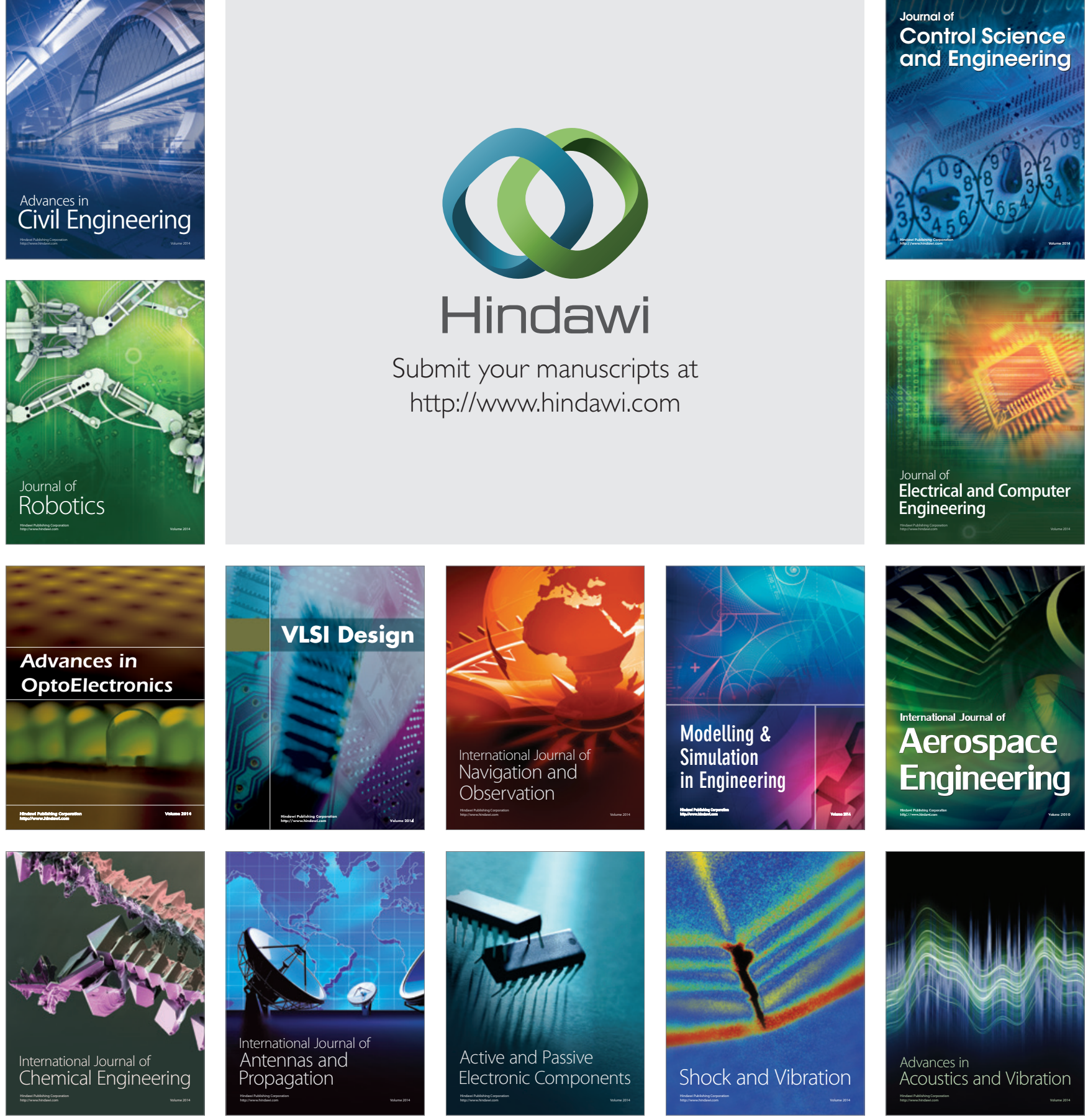